\title{
Accelerometer-Assessed Physical Activity and Sedentary Time in Youth With Disabilities
}

\author{
Karin Lobenius-Palmér, Birgitta Sjöqvist, Anita Hurtig-Wennlöf, \\ and Lars-Olov Lundqvist \\ Örebro University
}

This study compared accelerometer-assessed habitual physical activity (PA), sedentary time, and meeting PA recommendations among 102 youth with disabilities (7-20 years) in four subgroups-physical/visual impairments, intellectual disability, autism spectrum disorders, and hearing impairment — and 800 youth with typical development (8-16 years). Low proportions of youth with disabilities met PA recommendations, and they generally were less physically active and more sedentary than youth with typical development. The hearing impairment and autism spectrum disorder groups were the most and least physically active, respectively. Older age and to some extent female sex were related to less PA and more sedentary time. Considering the suboptimal levels of PA in youth with disabilities, effective interventions directed at factors associated with PA among them are needed.

Keywords: autism spectrum disorder, deaf/hearing impaired, health, intellectual disability, physical disabilities

The beneficial effects of physical activity (PA) on health for both children and adults are well known (Strong et al., 2005; World Health Organization [WHO],

\footnotetext{
(C) 2018 The Authors. Published by Human Kinetics, Inc. This is an Open Access article distributed under the terms of the Creative Commons Attribution License CC BY NC 4.0, which permits unrestricted noncommercial use, distribution, and reproduction in any medium, provided the original work is properly cited, the new use includes a link to the license, and any changes are indicated. See http://creativecommons.org/licenses/by-nc/4.0. This license does not cover any third-party material that may appear with permission in the article. For commercial use, permission should be requested from Human Kinetics, Inc., through the Copyright Clearance Center (http://www.copyright.com).

Lobenius-Palmér, Sjöqvist, and Lundqvist are with the University Health Care Research Center, Faculty of Medicine and Health, Örebro University, Örebro, Sweden. Hurtig-Wennlöf is with the School of Health Sciences, Faculty of Medicine and Health, Örebro University, Örebro, Sweden. Sjöqvist is now retired. Address author correspondence to Karin Lobenius-Palmér at karin.lobeniuspalmer@regionorebrolan.se.
} 
2010). In parallel, there is emerging evidence that sedentary behavior has an important negative influence on various health indicators (Dunstan, Howard, Healy, \& Owen, 2012; Tremblay et al., 2011). For individuals with disabilities, the ability to be physically active may be limited, increasing the risk for a sedentary lifestyle. Physical inactivity leads to deconditioning followed by even less PA, resulting in physical deterioration and increased difficulties in managing everyday activities. Furthermore, physical inactivity may lead to reduced societal interactions that ultimately lead to a decreased quality of life (Durstine et al., 2000; Fernhall \& Unnithan, 2002). Hence, it is important to increase PA and decrease sedentary time to ensure good present and future health for youth with disabilities.

To design PA interventions for youth with disabilities, it is first necessary to know levels of PA and how PA is influenced by different factors, such as sex, age, and type of disability. This information is important for researchers developing and refining interventions for increased PA and reduced sedentary time in youth with disabilities. It is also useful for rehabilitation professionals, who need to prioritize among different groups of youth, and for schoolteachers or recreation leaders to be able to maximize opportunities for PA throughout the day. In addition, this information can be important for public health planners and politicians customizing campaigns promoting increased PA and decreased sedentary time in this population.

To determine the level of PA among youth with disabilities and to identify subgroups of those with high and low PA and sedentary time, habitual PA and time spent in sedentary behavior should be quantified. Among the different methods available, accelerometry is advocated because it can quantify habitual PA and sedentary time in an objective, reliable, and practical way (Reilly et al., 2008). Accelerometry poses no demands on cognitive ability, making it particularly suitable for assessing PA and sedentary time in youth with disabilities.

To evaluate whether youth with disabilities are sufficiently active, assessment of whether they meet the PA recommendations of $\geq 60 \mathrm{~min} /$ day of moderate- to vigorous-intensity PA (MVPA) can be made. Previous accelerometer studies in youth with disabilities, however, show varying results regarding meeting these recommendations, such as between $0 \%$ and $25 \%$ of youth with cerebral palsy (Gorter, Noorduyn, Obeid, \& Timmons, 2012; Mitchell, Ziviani, \& Boyd, 2015b), between $0 \%$ and $50 \%$ of youth with intellectual disability (ID; Einarsson et al., 2015; Kozub, 2003; Roberts, 2007), between $23 \%$ and $100 \%$ of youth with autism spectrum disorders (ASDs; Bandini et al., 2013; Pan \& Frey, 2006; Rosser Sandt $\&$ Frey, 2005; Tyler, MacDonald, \& Menear, 2014), and between $21 \%$ and $93 \%$ of youth with Down syndrome (Esposito, MacDonald, Hornyak, \& Ulrich, 2012; Izquierdo-Gomez et al., 2014; Roberts, 2007; Shields, Dodd, \& Abblitt, 2009; Whitt-Glover, O'Neill, \& Stettler, 2006).

Given the large variation across studies and types of disabilities, it seems uncertain to what degree meeting PA recommendations differs across youth with different types of disabilities and to what extent meeting recommendations in these groups differs from that in youth with typical development (TD). Unfortunately, only one study presented data across groups of different disabilities (ID and Down syndrome) as well as youth with TD (Roberts, 2007). In addition, only a few studies have compared meeting recommendations in youth with disabilities with that in youth with TD. For instance, Einarsson et al. (2015) found that fewer youth with ID met the recommendations (0\%) than youth with TD (40\%), whereas Roberts (2007) 
found that youth with ID and youth with TD were rather similar (50\% and 43\%, respectively). Youth with Down syndrome in Roberts' (2007) study also met recommendations on a similar proportion (44\%). Furthermore, Rosser Sandt and Frey (2005) found that fewer youth with ASDs (67\%) than youth with TD (92\%) met the recommendations, whereas Bandini et al. (2013) found no difference between youth with ASDs (23\%) and youth with TD (43\%) and Tyler et al. (2014) found that all youth with ASDs (100\%) and youth with TD (100\%) met the recommendations. Hence, the results vary greatly across studies and types of disabilities and also vary across studies among youth with TD. It should be noted, however, that only Bandini et al. (2013) and Einarsson et al. (2015) statistically tested for group differences.

In addition to measures of meeting recommendations, accelerometer studies comparing habitual PA variables (e.g., average PA or MVPA) between youth with disabilities and youth with TD also show a pattern of varying results. That is, youth with cerebral palsy as well as youth with visual impairment were found to be less physically active than youth with TD (Houwen, Hartman, \& Visscher, 2009; Nooijen, Slaman, Stam, Roebroeck, \& Berg-Emons, 2014). Studies have reported lower levels of PA in youth with ID, Down syndrome, and ASDs than youth with TD (Einarsson et al., 2015; Tyler et al., 2014; Whitt-Glover et al., 2006), although other studies have not found any differences (Bandini et al., 2013; Roberts, 2007; Rosser Sandt \& Frey, 2005). Even higher PA levels among youth with intellectual disabilities than among youth with TD have been reported (Roberts, 2007).

Whether youth with disabilities are less, equally, or, in some cases, more physically active than youth with TD thus seems unclear. By comparing youth with different types of disabilities and youth with TD in the same study and with a similar protocol, a more reliable estimate of the variation across groups can be made.

With regard to sedentary time, previous accelerometer studies comparing youth with disabilities with youth with TD show that youth with disabilities such as cerebral palsy, visual impairment, or ASDs are more sedentary than youth with TD (Houwen et al., 2009; Nooijen et al., 2014, Obeid, Balemans, Noorduyn, Gorter, \& Timmons, 2014; Tyler et al., 2014). Youth with ID are reported to be both more sedentary than youth with TD (Einarsson et al., 2015) and similarly sedentary (Roberts, 2007). These studies indicate that youth with disabilities in general are more sedentary than youth with TD. However, because the number of studies is small and they cover only a few types of disabilities, one needs to be cautious in drawing any general conclusions regarding sedentary time. It is notable that youth with hearing impairment $(\mathrm{HI})$, although a significant group, are lacking in the accelerometer literature.

Factors other than type of disability, such as sex and age, may influence the levels of PA. However, few accelerometer studies have addressed these influences in youth with disabilities, limiting the knowledge of the impact of sex and age on the levels of PA.

Given the varying results from previous research, the aim of the present study was to investigate habitual PA, sedentary time, and meeting PA recommendations across several types of disabilities and in comparison with youth with TD. More specifically, the following research questions were addressed:

(a) How physically active and sedentary are youth with different disabilities compared with youth with TD? 
(b) How do PA and sedentary time vary with type of disability, sex, and age in youth with disabilities?

(c) To what extent do youth with disabilities meet current PA recommendations, and how does that compare with youth with TD?

(d) How does meeting PA recommendations vary with type of disability, sex, and age in youth with disabilities?

\section{Method}

The present study $(n=102)$ is part of a cross-sectional epidemiological study, Physical Activity and Health in Youth with Disabilities $(n=485)$, where different aspects of PA and health were assessed by self-report in youth with disabilities aged 7-20 years.

\section{Participants}

Participants were recruited from the Child and Youth Habilitation Centre (CYHC), Region Örebro County, Sweden. CYHC provides community-based rehabilitation and family support services for youth (aged 0-20 years) with various disabilities. All youth with disabilities requiring rehabilitation services in Örebro County are registered at CYHC. The inclusion criterion for the Physical Activity and Health in Youth with Disabilities study was age 7-20 years. In addition, inclusion criteria for the present study, which also included measurement of PA and sedentary time with an accelerometer, were being ambulatory (i.e., able to walk around a block) and not using mobility aids. An invitation to participate in this study was sent by mail to 1,296 individuals registered at CYHC. Sixteen individuals had moved, and one individual was deceased, so the invitation reached 1,279 individuals. Of these, 485 answered a questionnaire about different aspects of PA and health. Information on ambulatory ability and use of mobility aids was obtained via the questionnaire. A subsample of 138 individuals meeting the inclusion criteria of ambulation and no mobility aids agreed to participate in the present study. Among the volunteering youth, 24 individuals did not complete the accelerometer measurements because of refusal or problems wearing the accelerometer. Moreover, 8 participants' accelerometer registrations were considered invalid (for a definition, see below), and four participants' accelerometer registrations suffered technical problems and were excluded. The present study is based on accelerometer registrations from a total of 102 participants (59 males and 43 females).

Prior to the study, a power analysis was carried out showing that a total of 90 participants was necessary to reveal medium effect sizes in MVPA, given six independent variables in the regression analysis (Cohen, 1992). Based on that, a sample of 100 youth with disabilities was deemed appropriate.

Information on participants' diagnoses was obtained from medical records at CYHC. The participants had a variety of diagnoses, and more than half of the participants had two or more diagnoses. Participants' primary diagnoses were related to five types of impairments: physical impairment, visual impairment (one individual), ID, ASDs, and HI/deafness. Four disability groups were accordingly created, with physical and visual impairment merged into one group (Table 1). 
Table 1 Primary Diagnoses and Disability Groups

\begin{tabular}{lcl}
\hline $\begin{array}{l}\text { Disability } \\
\text { group }\end{array}$ & $\begin{array}{c}\text { Number of } \\
\text { participants }\end{array}$ & Primary diagnosis \\
\hline $\begin{array}{l}\text { Physical/visual } \\
\text { impairment }\end{array}$ & 5 & Cerebral palsy \\
$(n=16)$ & 3 & Neurofibromatosis \\
& 2 & Skeletal impairment \\
& 1 & Dystrophia myotonica \\
& 1 & Severe epilepsy \\
& 1 & $\begin{array}{l}\text { Unspecified congenital malformation of the nervous } \\
\text { system } \\
\text { Goldenhar syndrome } \\
\end{array}$ \\
& 1 & $\begin{array}{l}\text { Motor function disorder } \\
\text { Neonatal cerebral leukomalacia with visual } \\
\text { impairment } \\
\text { Mild, moderate, or unspecified ID (including four } \\
\text { participants with Down syndrome) } \\
\text { Autism spectrum disorder (without ID) }\end{array}$ \\
$\begin{array}{l}\text { ID } \\
\text { Autism spec- }\end{array}$ & 1 & Hearing impairment/deafness \\
trum disorder & 25 &
\end{tabular}

Note. ID = intellectual disability.

The physical/visual impairment group (PVI; $n=16$ ) consisted of participants with primary diagnoses related to physical or visual impairment, such as neurological and neuromuscular disorders (e.g., cerebral palsy, epilepsy, and dystrophia myotonica), skeletal impairments, congenital malformations and syndromes affecting multiple bodily functions (Goldenhar syndrome and neurofibromatosis), and visual impairment. Some diagnoses were unique to one participant. The majority of participants in the PVI group had one or more additional diagnoses, such as ID, ASD, HI, and speech or language impairment.

The ID group $(n=42)$ consisted of participants with mild, moderate, or unspecified ID as the primary diagnosis. Participants with Down syndrome were allocated to this group. About two thirds of the participants had one or more additional diagnoses, such as ASD, attention deficit hyperactivity disorder, HI, ventricular septum defect, tic disorder, or language impairment.

The ASD group $(n=25)$ consisted of participants with autism, Asperger syndrome, or unspecified pervasive developmental disorder as the primary diagnosis. About half of the participants had one or more additional diagnoses, such as attention deficit hyperactivity disorder, attention deficit disorder without hyperactivity, speech or language impairment, or tic disorder. None had physical impairments, ID, or HIs.

Finally, the HI group $(n=19)$ consisted of participants with HI or deafness. One participant had an additional diagnosis of sleep disorder. None had physical or visual impairments, ID, or ASDs. 
The regional ethical review board in Uppsala, Sweden, approved the study. Participant assent and parental written informed consent to the research were obtained from all participants. Participants aged 18 years or older provided their own written informed consent whenever possible.

To compare the PA and sedentary time of youth with disabilities with that of youth with TD, accelerometer data from a reference group of youth with TD from a previous study were used; hereafter, this is referred to as the TD group. The TD group consisted of 800 youth (356 males and 444 females) in two age groups (8-10 years and 14-16 years) from the Swedish component of the European Youth Heart Study (EYHS). EYHS was a school-based cross-sectional study of youth with TD, where PA and sedentary time were measured using an Actigraph accelerometer (model 7164; ActiGraph LLC, Pensacola, FL) in the same way as used with the youth with disabilities in the present study. Study design, selection criteria, and basic characteristics of the participants in the EYHS are reported elsewhere (Hurtig-Wennlöf, Yngve, Nilsson \& Sjöström, 2005; Wennlöf, Yngve, \& Sjöström, 2003).

\section{Instrumentation}

Habitual PA and sedentary time in youth with disabilities were measured with the Actigraph activity monitor (model GT1M; Actigraph LLC). The Actigraph is the most widely used activity monitor and has the largest body of evidence supporting its feasibility, reliability, and validity in children with TD (de Vries, Bakker, Hopman-Rock, Hirasing, \& van Mechelen, 2006; Riddoch et al., 2004; Trost, 2007). It has also been shown to be feasible, reliable, and valid for youth with cerebral palsy (Clanchy, Tweedy, Boyd, \& Trost, 2011; Gorter et al., 2012), ASDs (Rosser Sandt \& Frey, 2005), and ID and Down syndrome (Roberts, 2007).

The Actigraph-GT1M is a uniaxial accelerometer that records vertical accelerations, which are processed to produce "counts" over a time period (epoch; Chen \& Bassett, 2005). Data in the present study were further analyzed with the custom-written software MAHUffe (Medical Research Council, Epidemiology Unit, Univeristy of Cambridge, UK, 2015). An epoch length of $60 \mathrm{~s}$, the same as in the EYHS, was used in the present study. Wear time validation was carried out, where continuous periods of zero counts exceeding $20 \mathrm{~min}$ were regarded as "accelerometer not worn" and not included in the registered time.

At least 3 days of valid recordings with $\geq 600 \mathrm{~min} /$ day served as the criterion for a valid registration. This criterion is in line with the EYHS and previous studies in youth using the Actigraph (Andersen et al., 2006; Hurtig-Wennlöf, 2005; Mattocks et al., 2008).

Outcome variables derived from the accelerometer data were (a) average PA, (b) time spent in light-intensity PA (LPA), (c) time spent in MVPA, (d) sedentary time, and (e) meeting PA recommendations (yes or no). Average PA was calculated by dividing total counts during a day with total minutes of registered time that day (expressed in counts per minute [cpm]). To categorize time spent (min/day) in the different PA intensities, LPA, MVPA, and sedentary, accelerometer count cut points were used. For sedentary, the cut point was $<100 \mathrm{cpm}$ (Kim, Lee, Peters, Gaesser, \& Welk, 2014). For LPA and MVPA, age-specific count cut points for youth (Trost et al., 2002; participants aged 7-18 years) and adults (Freedson, 
Melanson, \& Sirard, 1998; participants aged 19-20 years) were used. The sedentary cut point and age-specific cut points for youth (Trost et al., 2002) used for participants aged 7-18 years were the same as used for youth with TD.

The PA recommendations used in this study are those from the World Health Organization (WHO, 2010). WHO recommends that children (aged 5-17 years) accumulate at least 60 min of MVPA per day. WHO further recommends that adults (aged 18-64 years) accumulate at least 150 min of moderate-intensity PA or 75 min of vigorous-intensity PA, or an equivalent combination of moderate- and vigorous-intensity activity per week. WHO's child recommendations were applied to participants aged 7-17 years, and its adult recommendations to participants aged 18-20 years.

\section{Procedure}

The invitation to participate was sent together with a questionnaire to each youth's home. The invitation included a description of the study in easy-to-read language. For youth under 18 years of age, a parent or legal guardian was asked to give written consent and to read the description to the youth if the youth was not able to read in order to obtain his or her assent. The questionnaire was completed by the youth with assistance from an adult if necessary. Information derived from the questionnaire used in this study concerned ambulatory ability and use of mobility aids.

The accelerometer was initialized and sent to the participant by mail. Participants and parents were given verbal and written instructions on wearing the accelerometer in an elastic belt around the waist, at the center of the back (Yngve, Nilsson, Sjostrom, \& Ekelund, 2003), for seven consecutive days during all waking hours except during water activities. After 7 days, the accelerometer was returned by mail and the data were downloaded. Participants and parents were also asked to note wearing and nonwearing times, as well as details about the nonwearing times, in an activity $\log$. The log was then compared with visually inspected accelerometer data to ensure consistency between recorded data and actual wearing time. The activity $\log$ also served as a reminder to increase compliance and to monitor reasons for not wearing the accelerometer.

\section{Statistical Analyses}

Statistical analyses were performed with IBM SPSS Statistics, version 22.0 (IBM Corp., Armonk, NY). Before analyses, average PA was logarithmically transformed (natural logarithm) to obtain normal distribution. However, untransformed values are presented in Table 2 for clarity. No other variables were in need of transformation.

The data regarding sex (dichotomous; male or female), age (continuous; 7-20 years), and registered time (continuous; 622-1,106 min/day) were unevenly distributed across study groups. To adjust for possible bias and as recommended by Altman (1991), multiple linear regression analyses and multiple logistic regression analyses were undertaken. In both the multiple linear regression analyses and the multiple logistic regression analyses, all independent variables were entered together (i.e., we used the "enter" method in SPSS). 


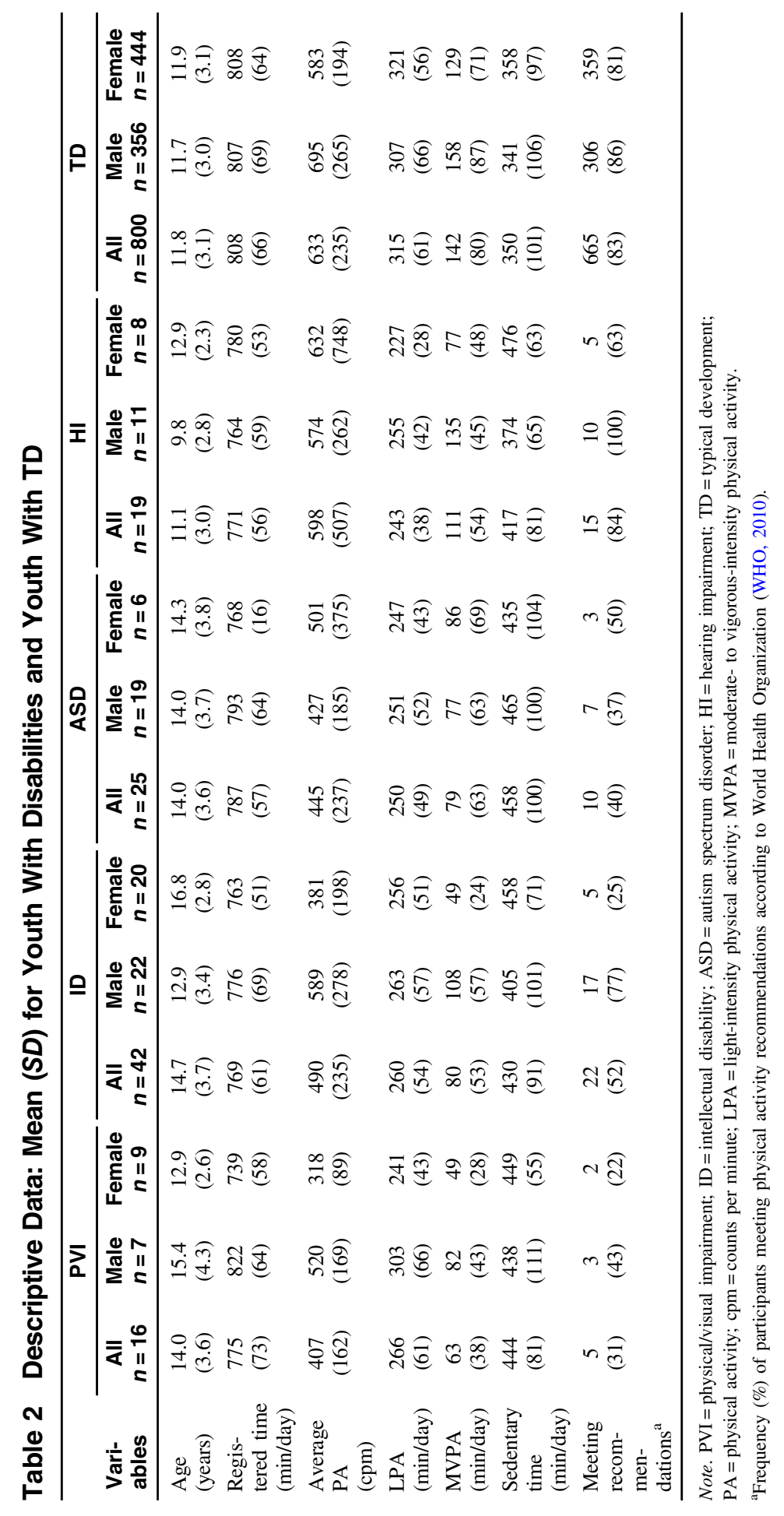


In reference to our research questions, to investigate how PA (i.e., average PA, LPA, and MVPA) and sedentary time vary with type of disability, sex, and age in youth with disabilities, we performed multiple regression analyses, with the four disability groups (dummy-coded), sex, age, and registered time serving as the independent variables and average PA, LPA, MVPA, and sedentary time serving as the dependent variables. Again four separate multiple regression analyses were performed. Thereafter, we investigated how physically active and sedentary were youth with different disabilities compared with youth with TD. To investigate this, we performed multiple regression analyses with all study groups (i.e., the four disability groups and the group with TD; dummy-coded), sex, age, and registered time (independent variables) and average PA, LPA, MVPA, and sedentary time (dependent variables). Again, four separate multiple regression analyses were performed, one for each dependent variable. The results from the multiple regression analyses were expressed in the regression coefficient $B$ and its standard error ( $S E$ ), as well as the standardized $\beta$ and the $p$ value.

To investigate how meeting PA recommendations vary with type of disability, sex, and age in youth with disabilities and to what extent youth with disabilities meet current PA recommendations compared with youth with TD, we used multiple logistic regression analyses. The dichotomous "meeting PA recommendations" variable (i.e., the dependent variable) was regressed on study groups, sex, age, and registered time (i.e., the independent variables) in the same way as for the multiple regression analysis mentioned above. The results from the logistic regression were expressed in odds ratios (ORs) and their 95\% confidence intervals (CIs). In all analyses, the level of statistical significance was set at $p<.05$.

\section{Results}

\section{Accelerometer Measurement}

Regarding accelerometry, $7 \%$ of the 102 participants in the present study provided 3 days of valid recordings and $93 \%$ provided 4-7 days. Although a weekend day was not specified as necessary to fulfill validity criteria, $87 \%$ of the 102 registrations comprised both weekdays and weekend days. All other descriptive data for the PA variables for the disability groups and the TD group are shown in Table 2.

\section{Average PA, LPA, MVPA, and Sedentary Time}

Average PA. As shown in Table 3, each of the four disability groups had significantly lower levels of average PA than the TD group. There was no statistically significant difference, however, in average PA across disability groups. In regard to sex, the analyses showed that in the ID and TD groups, males had higher average PA than females $(B=0.34, S E=0.14, \beta=0.43$, $p<.05$ and $B=0.15, S E=0.023, \beta=0.20, p<.001$, respectively). In regard to age, there was no significant influence of age on average PA in any of the four disability groups; however, the older the youth in the TD group, the lower the average PA $(B=-0.06, S E=0.004, \beta=-0.45, p<.001)$. 
Table 3 Multiple Regression Analysis Results for PA Variables Regressed on Youth With Disabilities and Youth With TD, Adjusted for Sex, Age, and Registered Time Biases

\begin{tabular}{lcccccc}
\hline Dependent & Independent & $\boldsymbol{B}$ & SE & $\boldsymbol{\beta}$ & $\boldsymbol{t}$ & $\boldsymbol{p}$ \\
\hline Average PA (cpm) & TD & [Reference] & & & & \\
& PVI & -0.32 & 0.09 & -0.11 & -3.37 & .001 \\
& ID & -0.13 & 0.06 & -0.07 & -2.27 & .023 \\
& ASD & -0.32 & 0.07 & -0.13 & -4.56 & $<.001$ \\
LPA (min/day) & HI & -0.21 & 0.08 & -0.08 & -2.65 & .008 \\
& TD & [Reference] & & & & \\
& PVI & -26.55 & 12.51 & -0.06 & -2.12 & .034 \\
& ID & -29.82 & 8.06 & -0.10 & -3.70 & .001 \\
MVPA (min/day) & ASD & -46.81 & 10.14 & -0.12 & -4.62 & $<.001$ \\
& HI & -51.41 & 11.45 & -0.12 & -4.49 & $<.001$ \\
& TD & {$[$ Reference $]$} & & & & \\
& PVI & -33.58 & 12.75 & -0.06 & -2.63 & .009 \\
& ID & -4.25 & 8.21 & -0.01 & -0.52 & .605 \\
& ASD & -26.25 & 10.34 & -0.05 & -2.54 & .011 \\
& HI & -43.22 & 11.67 & -0.08 & -3.70 & $<.001$ \\
& TD & {$[$ Reference] } & & & & \\
& PVI & 60.13 & 17.54 & 0.08 & 3.43 & .001 \\
& ID & 34.07 & 11.30 & 0.07 & 3.02 & .003 \\
& ASD & 73.06 & 14.23 & 0.12 & 5.14 & $<.001$ \\
& HI & 94.63 & 16.06 & 0.13 & 5.89 & $<.001$ \\
\hline
\end{tabular}

Note. $S E=$ standard error of $B ; \mathrm{PA}=$ physical activity; $\mathrm{cpm}=$ counts per minute; $\mathrm{TD}=$ typical development; $\mathrm{PVI}=$ physical/visual impairment; $\mathrm{ID}=$ intellectual disability; $\mathrm{ASD}=$ autism spectrum disorder; $\mathrm{HI}=$ hearing impairment; LPA = light-intensity PA; MVPA = moderate- to vigorousintensity PA.

Light-Intensity PA. As shown in Table 3, each of the four disability groups spent significantly less time in LPA than the TD group. However, there was no statistically significant difference across disability groups. There were no significant influences of sex or age on LPA in any of the four disability groups. In the TD group, males had less LPA compared with females $(B=-13.59, S E=3.48, \beta=-0.11, p<.001)$, and the older the youth, the less the LPA $(B=-1.61, S E=0.59, \beta=-0.81, p<.01)$.

Moderate- to Vigorous-Intensity PA. As shown in Table 3, all groups but the ID group spent significantly less time in MVPA than the TD group. However, there was no statistically significant difference across disability groups. In regard to sex, the analyses showed that in the PVI and TD groups, males had more MVPA than females $(B=35.79, S E=14.29, \beta=0.49, p<.05$ and $B=26.20, S E=3.55, \beta=$ $0.16, p<.001$, respectively). There was a significant influence of age on MVPA in all four disability groups as well as in the TD group. The older the youth, the lesser 
the time spent in MVPA: PVI $(B=-8.37, S E=1.84, \beta=-0.79, p<.001)$, ID $(B=$ $-10.12, S E=1.69, \beta=-0.70, p<.001)$, ASD $(B=-9.39, S E=3.18, \beta=-0.54$, $p<.01)$, HI $(B=-12.25, S E=3.34, \beta=-0.67, p<.01)$, and TD $(B=-20.45$, $S E=0.60, \beta=-0.79, p<.001)$.

Sedentary Time. As shown in Table 3, each of the four disability groups spent significantly more time sedentary than the TD group. However, there was no statistically significant difference across disability groups. In regard to sex, the analyses showed that in the HI and TD groups, males had less sedentary time than females $(B=-52.23, S E=22.97, \beta=-0.33, p<.05$ and $B=-12.61, S E=4.81$, $\beta=-0.06, p<.01$, respectively). There was a significant influence of age on sedentary time, with older youth spending more time sedentary than younger youth in the ID group $(B=10.32, S E=3.54, \beta=0.41, p<.01)$, in the HI group $(B=13.22, S E=$ $22.97, \beta=0.6348, p<.01)$, and in the TD group $(B=22.07, S E=0.81, \beta=0.67$, $p<.001)$.

\section{Meeting WHO's PA Recommendations}

Proportions of the youth in the disability groups and the TD group meeting WHO's PA recommendations are shown in Table 2. Fewer in the PVI and ASD groups met recommendations than in the TD group (Table 4). When looking at the four disability groups only, the odds of meeting recommendations were significantly lower in the PVI group than in the HI group $(O R=0.13,95 \% \mathrm{CI}=0.02-$ $0.88)$ and lower in the ASD group than in the ID and HI groups $(O R=0.21,95 \%$ $\mathrm{CI}=0.05-0.89$ and $O R=0.13,95 \% \mathrm{CI}=0.02-0.86$, respectively). There was no significant influence of sex in any of the four disability groups. Regarding age, the OR of meeting recommendations was significantly lower with increasing age in the ID group $(O R=0.59,95 \% \mathrm{CI}=0.41-0.85)$ and in the ASD group $(O R=0.65$, $95 \% \mathrm{CI}=0.46-0.93$ ), demonstrating that the older the youth, the fewer in these groups met PA recommendations. In the TD group, males and females met recommendations to the same extent; regarding age, however, the older the youth, the fewer met the recommendations $(O R=0.54,95 \% \mathrm{CI}=0.48-0.61)$.

\section{Table 4 Multiple Logistic Regression Analysis Results for Meeting PA Recommendations Among Youth With Disabilities, With Youth With TD Serving as Reference}

\begin{tabular}{lccc}
\hline Groups & OR & 95\% Cl of $\mathbf{O R}$ & $\boldsymbol{p}$ \\
\hline TD & [Reference] & & \\
PVI & 0.11 & $0.03-0.42$ & .001 \\
ID & 0.75 & $0.31-1.80$ & .514 \\
ASD & 0.15 & $0.05-0.47$ & .001 \\
HI & 0.89 & $0.19-4.23$ & .878 \\
\hline
\end{tabular}

Note. The results are adjusted for sex, age, and registered time biases. $\mathrm{PA}=$ physical activity; $\mathrm{OR}=$ odds ratio; $\mathrm{CI}=$ confidence interval; $\mathrm{TD}=$ typical development; $\mathrm{PVI}=$ physical/visual

impairment; $\mathrm{ID}=$ intellectual disability; $\mathrm{ASD}=$ autism spectrum disorder; $\mathrm{HI}=$ hearing impairment. 


\section{Discussion}

The major findings of this study were that youth with disabilities generally were less physically active and more sedentary than youth with TD. As few as a third of the youth in the PVI group, in contrast to eight out of 10 youth with TD, met PA recommendations. The results thus indicate that many youth with disabilities may be at higher risk of not reaching levels of PA, suggested to be health enhancing, than youth with TD.

The lower habitual PA (except MVPA for the ID group) and higher sedentary time than in the TD group in the current study are consistent with previous accelerometer studies comparing youth with different disabilities and youth with TD in Europe and North America (Einarsson et al., 2015; Houwen et al., 2009; Nooijen et al., 2014; Obeid et al., 2014; Tyler et al., 2014). Furthermore, the finding that a low proportion of youth with disabilities met the PA recommendations is in line with previous findings in youth with various disabilities across the Western world (Bandini et al., 2013; Einarsson et al., 2015; Esposito et al., 2012; Gorter et al., 2012; Izquierdo-Gomez et al., 2014; Kozub, 2003; Mitchell et al., 2015b; Shields et al., 2009), indicating that low PA and high sedentary time in youth with disabilities is a widespread phenomenon, at least in developed countries.

Although youth with disabilities were less active than youth with TD in almost all PA variables, there were some exceptions. There was, for instance, no difference between the ID and TD groups in MVPA and meeting recommendations, which is in keeping with results from accelerometer studies by Roberts (2007) and Whitt-Glover et al. (2006).

Given the varying levels of PA across disability groups reported in previous research, the lack of differences in habitual PA and sedentary time across disability groups was somewhat unexpected. However, some differences were found. The proportion meeting recommendations differed across disability groups, with the HI group meeting recommendations to a higher extent than both the PVI and ASD groups; the ID group was intermediate. This difference has not been described in previous accelerometer studies, but is in line with previous studies using pedometer and self-report, showing that youth with HI were among the most physically active, youth with ID were somewhat less active, and youth with physical impairments were least active (Longmuir \& Bar-Or, 2000; Sit, Lindner, \& Sherrill, 2002; Suzuki et al., 1991). In addition, the ASD group met recommendations to a lower degree than both the ID and the HI groups, as well as to a lower degree than the TD group. This is in line with previous results showing that youth with ASDs met recommendations to a lower degree than youth with TD (Rosser Sandt \& Frey, 2005) and indicates that the presence of ASD may be a risk marker for low PA and that youth with ASD should be a particular focus of PA-enhancing interventions.

Influences of sex were found within three out of four disability groups. In the PVI group, males had higher PA than females. This is in line with findings on youth with cerebral palsy (Mitchell, Ziviani, \& Boyd, 2015a, 2015b), which is a diagnosis included in the PVI group. Moreover, males in the ID group were more physically active than females in this group, which has been noted in individuals with ID elsewhere (Phillips \& Holland, 2011). Higher pedometer scores for males 
with ID have also been reported (Suzuki et al., 1991). Not reported previously, males with HI were less sedentary than females with HI. The only group in which no sex difference was found was the ASD group. This suggests that youth with ASD have a somewhat different PA pattern from that in youth in other disability groups and youth with TD. The finding that males are more physically active than females, as found in the PVI, ID, and HI groups, is in line with results for youth with TD from the current study and previous studies (Matthews et al., 2008; Riddoch et al., 2004; Trost et al., 2002). Thus, one might wonder if there are similar mechanisms behind the sex differences in youth with disabilities and those in youth with TD. This topic is not fully explored in this population and needs to be investigated further.

The influence of age on PA was large among youth with disabilities and in line with previous studies on youth with cerebral palsy (Mitchell et al., 2015a), Down syndrome (Esposito et al., 2012; Shields et al., 2009), visual impairment (Ayvazoglu, Oh, \& Kozub, 2006; Kozub \& Oh, 2004), ID (Kozub, 2003), ASDs (Pan \& Frey, 2005, 2006), and on youth with TD (Riddoch et al., 2004; Trost et al., 2002). Particularly, time spent in intense PA (i.e., MVPA and meeting PA recommendations) decreased with age, whereas no influence of age was found on average PA or on time spent in less intense PA (i.e., LPA). The finding that PA declined with age in more intense PA but not in less intense PA has been reported in a study on youth with the Down syndrome (Izquierdo-Gomez et al., 2014) showing that MVPA and meeting PA recommendations decreased with age whereas LPA and average PA did not.

Parallel to the age-related decline in MVPA and meeting PA recommendations, the older the youth in the ID and HI groups, the more the time spent sedentary. This is consistent with previous findings on youth with cerebral palsy, Down syndrome (Esposito et al., 2012; Izquierdo-Gomez et al., 2014), and youth with TD in the current study as well as previous studies (Matthews et al., 2008).

\section{Implications}

Current PA recommendations are based on time spent in MVPA (WHO, 2010). However, for youth with disabilities, various factors may impede the ability to achieve 60 min of MVPA per day. Time spent in MVPA only accounts for a small proportion of the day. The rest of the day is spent in LPA and sedentary behavior. Considering the health benefits of LPA (Carson et al., 2013; Healy et al., 2008) and the unfavorable effects of sedentary behavior (Dunstan et al., 2012; Tremblay et al., 2011), reallocating time spent being sedentary into time spent in LPA would be favorable. In the reallocation process, it is important to consider all potential areas where sedentary behavior can be decreased during the whole day, such as during transport to school, during the whole school day, and at home in the evening.

Because patterns of PA and sedentary behavior are carried through into adulthood (Telama et al., 2005), it is important to ensure that positive PA habits are established early in life and to promote positive PA habits to counteract decline in PA as children grow older. Regarding influence of sex, female youth with disabilities have among the lowest levels of PA. This suggests that interventions should target methods for increasing PA in females. 


\section{Strengths and Limitations}

The strengths of the study were the use of objective measures to assess habitual PA and sedentary time as well as the use of a single assessment protocol, which allows for direct comparisons across disability groups and youth with TD. However, there is currently a lack of consensus in the literature on accelerometer practice, especially in regard to youth with disabilities. Therefore, and for comparability with the EYHS, we chose one of the most widely used accelerometers (the Actigraph; de Vries et al., 2006; Trost, 2007), one of the most widely used epochs (60 s; Cain, Sallis, Conway, Van Dyck, \& Calhoon, 2013), and one of the most widely used accelerometer count cut points (Cain et al., 2013).

Some limitations should be noted. First, the youth who volunteered for this study might have been those who were more physically active than the nonparticipating ones. As a consequence, this bias might have resulted in smaller differences between youth with disability and youth with TD. The differences in the population may thus be larger than observed in the present sample.

Second, youth with disability generally have a diversity of diagnoses, abilities, and behaviors that makes it difficult to form homogeneous groups. We categorized the individuals into rather homogeneous groups according to the primary diagnosis, yet few differences in PA were found across the four disability groups. This result implies a larger variability in PA within the groups than across the groups. It should be noted that the variability within the disability groups was similar to the variability within the TD group. However, to further evaluate differences between different types of disabilities, studies using larger populations are needed. Future research may also address how habitual PA and sedentary time vary over time, such as with age, which calls for studies with longitudinal designs.

Third, the total sample size was acceptable and among the larger found in PA research on youth with disabilities using objective methods. However, when dividing the sample into subgroups, the subgroup sample sizes were on the small side. This is particularly problematic when dividing the sample further into sex within disability groups. For this reason, caution is warranted regarding conclusions about influences of sex among youth with disabilities due to the small number of either females or males in some of the groups. More reliable results regarding sex differences across disability groups warrant studies using larger sample sizes.

Fourth, the interval for sampling the accelerometer counts (epoch) used in the present study was $60 \mathrm{~s}$. Using this epoch for children and adolescents has been questioned because children's patterns of PA have been described as highly intermittent (Bailey et al., 1995). Thus a shorter epoch might have more accurately assessed high-intensity activity (Edwardson \& Gorely, 2010; Nilsson, Ekelund, Yngve, \& Sjöström, 2002; Reilly et al., 2008; Rowlands, Powell, Humphries, \& Eston, 2006). However, previous studies found that the choice of epoch length primarily affected vigorous-intensity activity, not MVPA (where vigorousintensity activity is integrated with moderate-intensity activity). Because vigorous-intensity activity was not examined separately in the present study, but rather MVPA, the 60-s epoch used in the present study was considered adequate. 
Furthermore, because it is recommended not to use different epochs when comparing different studies (Edwardson \& Gorely, 2010), and because the previously collected data for the TD group used a 60-s epoch, it was important to use the same epoch in the present study. Potential underestimations of PA would then be the same for both the study and the TD group using the same epoch.

Fifth, while activity monitoring lasting 4-5 days has been suggested for reliable estimates of daily PA in youth (Trost, Pate, Freedson, Sallis, \& Taylor, 2000 ), the current study used at least 3 days of valid recordings with $\geq 600$ $\mathrm{min} /$ day as the criterion for a valid registration. This criterion is in line with the EYHS and with previous studies in youth using the Actigraph (Andersen et al., 2006; Hurtig-Wennlöf, 2005; Mattocks et al., 2008). Notably, of the 102 participants in the present study, $7 \%$ provided 3 days of valid recordings and 93\% provided $4-7$ days.

Finally, it has been suggested that population-specific cut points and energy-expenditure prediction formulas should be used because individuals with disabilities may display different economy of movement (Clanchy et al., 2011; Fernhall \& Unnithan, 2002; Frey, Stanish, \& Temple, 2008). There is no consensus, however, on which cut points to use, either for a whole population with different disabilities or across different disabilities. Therefore, we chose the most widely used cut points. These cut points seemed reasonable for this group because average PA, which is not affected by cut points, was largely mirrored by sedentary time, LPA, and MVPA. Future research may conclude that different cut points are more appropriate for youth with disabilities. Our study thus presented measurement variables that were based on cut points and those that were not, allowing for comparisons across different measurement variables.

The few differences across the disability groups indicate that the presence of any disability may constitute a risk for low PA and high sedentary time. Although the disability itself may serve as a risk marker, there are a number of other operating factors affecting PA, such as individual abilities, habits, social networks, living conditions, and physical environment. Investigating possible correlates of PA and sedentary time other than age and sex was beyond the scope of the present study. It is, however, an important topic for future research because finding factors associated with PA in youth with disabilities enables the development of effective interventions.

\section{Conclusions}

The results from the present study show that youth with disabilities are not sufficiently physically active and are highly sedentary, which means that they are subject to a risk of poor health. This prompts the development of various strategies and interventions, aiming to increase PA and/or decrease sedentary time. Considering the suboptimal levels of PA, including sedentary time, found among youth with disabilities, these actions should include all youth with disabilities. However, as indicated by the current study results, they should also be targeted specifically to youth with ASDs as well as females and both younger and older individuals to counteract an age decline in PA. 


\section{Acknowledgments}

We wish to thank the youth and their parents for their participation in the study. For their financial support, we also thank the Royal Wedding Fund, the National Association for Disabled Children, and the Adolescent Research Foundation-RBU's Research Foundation; the Sunnerdahl Handikappfond; Odd Fellows, Örebro; and the Centre for Rehabilitation Research and Research Committee at Region Örebro County.

\section{References}

Altman, D.G. (1991). Practical statistics for medical research. London, UK: Chapman \& Hall.

Andersen, L.B., Harro, M., Sardinha, L.B., Froberg, K., Ekelund, U., Brage, S., \& Anderssen, S.A. (2006). Physical activity and clustered cardiovascular risk in children: A cross-sectional study (The European Youth Heart Study). Lancet, 368, 299-304. PubMed doi:10.1016/S0162-0908(08)70100-4

Ayvazoglu, N.R., Oh, H.-K., \& Kozub, F.M. (2006). Explaining physical activity in children with visual impairments: A family systems approach. Exceptional Children, 72, 235. doi:10.1177/001440290607200207

Bailey, R.C., Olson, J., Pepper, S.L., Porszasz, J., Barstow, T.J., \& Cooper, D.M. (1995). The level and tempo of children's physical activities: An observational study. Medicine \& Science in Sports \& Exercise, 27, 1033-1041. PubMed doi:10.1249/ 00005768-199507000-00012

Bandini, L.G., Gleason, J., Curtin, C., Lividini, K., Anderson, S.E., Cermak, S.A., ... Must, A. (2013). Comparison of physical activity between children with autism spectrum disorders and typically developing children. Autism, 17, 44-54. PubMed doi: $10.1177 / 1362361312437416$

Cain, K.L., Sallis, J.F., Conway, T.L., Van Dyck, D., \& Calhoon, L. (2013). Using accelerometers in youth physical activity studies: A review of methods. Journal of Physical Activity \& Health, 10, 437-450. PubMed doi:10.1123/jpah.10.3.437

Carson, V., Ridgers, N.D., Howard, B.J., Winkler, E.A., Healy, G.N., Owen, N., ... Salmon, J. (2013). Light-intensity physical activity and cardiometabolic biomarkers in US adolescents. PLOS ONE, 8, e71417. PubMed doi:10.1371/journal.pone.0071417

Chen, K.Y., \& Bassett, D.R., Jr. (2005). The technology of accelerometry-based activity monitors: Current and future. Medicine \& Science in Sports \& Exercise, 37(Suppl. 11), S490-S500. PubMed doi:10.1249/01.mss.0000185571.49104.82

Clanchy, K.M., Tweedy, S.M., Boyd, R.N., \& Trost, S.G. (2011). Validity of accelerometry in ambulatory children and adolescents with cerebral palsy. European Journal of Applied Physiology, 111, 2951-2959. PubMed doi:10.1007/s00421-011-1915-2

Cohen, J. (1992). A power primer. Psychological Bulletin, 112, 155-159. PubMed doi:10.1037/0033-2909.112.1.155

de Vries, S.I., Bakker, I., Hopman-Rock, M., Hirasing, R.A., \& van Mechelen, W. (2006). Clinimetric review of motion sensors in children and adolescents. Journal of Clinical Epidemiology, 59, 670-680. PubMed doi:10.1016/j.jclinepi.2005.11.020

Dunstan, D.W., Howard, B., Healy, G.N., \& Owen, N. (2012). Too much sitting-A health hazard. Diabetes Research and Clinical Practice, 97, 368-376. PubMed doi:10.1016/ j.diabres.2012.05.020

Durstine, J.L., Painter, P., Franklin, B.A., Morgan, D., Pitetti, K.H., \& Roberts, S.O. (2000). Physical activity for the chronically ill and disabled. Sports Medicine, 30, 207-219. PubMed doi:10.2165/00007256-200030030-00005 
Edwardson, C.L., \& Gorely, T. (2010). Epoch length and its effect on physical activity intensity. Medicine \& Science in Sports \& Exercise, 42, 928-934. PubMed doi:10.1249/MSS.0b013e3181c301f5

Einarsson, I.O., Olafsson, A., Hinriksdottir, G., Johannsson, E., Daly, D., \& Arngrimsson, S.A. (2015). Differences in physical activity among youth with and without intellectual disability. Medicine \& Science in Sports \& Exercise, 47(2), 411-418. PubMed doi:10.1249/mss.0000000000000412

Esposito, P.E., MacDonald, M., Hornyak, J.E., \& Ulrich, D.A. (2012). Physical activity patterns of youth with Down syndrome. Intellectual and Developmental Disabilities, 50, 109-119. PubMed doi:10.1352/1934-9556-50.2.109

Fernhall, B., \& Unnithan, V.B. (2002). Physical activity, metabolic issues, and assessment. Physical Medicine and Rehabilitation Clinics of North America, 13, 925-947. PubMed doi:10.1016/S1047-9651(02)00031-1

Freedson, P.S., Melanson, E., \& Sirard, J. (1998). Calibration of the Computer Science and Applications, Inc. accelerometer. Medicine \& Science in Sports \& Exercise, 30, $777-$ 781. PubMed doi:10.1097/00005768-199805000-00021

Frey, G.C., Stanish, H.I., \& Temple, V.A. (2008). Physical activity of youth with intellectual disability: Review and research agenda. Adapted Physical Activity Quarterly, 25, 95-117. PubMed doi:10.1123/apaq.25.2.95

Gorter, J.W., Noorduyn, S.G., Obeid, J., \& Timmons, B.W. (2012). Accelerometry: A feasible method to quantify physical activity in ambulatory and nonambulatory adolescents with cerebral palsy. International Journal of Pediatrics, 2012, 329284. PubMed doi:10.1155/2012/329284

Healy, G.N., Wijndaele, K., Dunstan, D.W., Shaw, J.E., Salmon, J., Zimmet, P.Z., \& Owen, N. (2008). Objectively measured sedentary time, physical activity, and metabolic risk: The Australian Diabetes, Obesity and Lifestyle Study (AusDiab). Diabetes Care, 31, 369-371. PubMed doi:10.2337/dc07-1795

Houwen, S., Hartman, E., \& Visscher, C. (2009). Physical activity and motor skills in children with and without visual impairments. Medicine \& Science in Sports \& Exercise, 41, 103-109. PubMed doi:10.1249/MSS.0b013e318183389d

Hurtig Wennlöf, A. (2005). Cardiovascular risk factors in children (Doctoral thesis). Karolinska Institute, Stockholm, Sweden.

Hurtig-Wennlöf, A., Yngve, A., Nilsson, T.K., \& Sjöström, M. (2005). Serum lipids, glucose and insulin levels in healthy schoolchildren aged 9 and 15 years from Central Sweden: Reference values in relation to biological, social and lifestyle factors. Scandinavian Journal of Clinical \& Laboratory Investigation, 65, 65-76. PubMed doi:10.1080/00365510410003110

IBM Corp. (2013). IBM SPSS Statistics for Windows (Version 22.0) [Computer software]. Armonk, NY: IBM Corp.

Izquierdo-Gomez, R., Martinez-Gomez, D., Acha, A., Veiga, O.L., Villagra, A., \& DiazCueto, M. (2014). Objective assessment of sedentary time and physical activity throughout the week in adolescents with Down syndrome. The UP\&DOWN study. Research in Developmental Disabilities, 35, 482-489. PubMed doi:10.1016/ j.ridd.2013.11.026

Kim, Y., Lee, J.M., Peters, B.P., Gaesser, G.A., \& Welk, G.J. (2014). Examination of different accelerometer cut-points for assessing sedentary behaviors in children. PLoS ONE, 9, e90630. PubMed doi:10.1371/journal.pone.0090630

Kozub, F.M. (2003). Explaining physical activity in individuals with mental retardation: An exploratory study. Education and Training in Developmental Disabilities, 38, 302-313.

Kozub, F.M., \& Oh, H.-K. (2004). An exploratory study of physical activity levels in children and adolescents with visual impairments. Clinical Kinesiology, 58(3), 1-7. 
Longmuir, P.E., \& Bar-Or, O. (2000). Factors influencing the physical activity levels of youths with physical and sensory disabilities. Adapted Physical Activity Quarterly, 17, 40-53. doi:10.1123/apaq.17.1.40

Matthews, C.E., Chen, K.Y., Freedson, P.S., Buchowski, M.S., Beech, B.M., Pate, R.R., \& Troiano, R.P. (2008). Amount of time spent in sedentary behaviors in the United States, 2003-2004. American Journal of Epidemiology, 167, 875-881. PubMed doi:10.1093/aje/kwm390

Mattocks, C., Deere, K., Leary, S., Ness, A., Tilling, K., Blair, S.N., \& Riddoch, C. (2008). Early life determinants of physical activity in 11 to 12 year olds: Cohort study. British Journal of Sports Medicine, 42, 721-724. PubMed doi:10.1136/ bmj.39385.443565.BE

Medical Research Council, Epidemiology Unit, Univeristy of Cambridge, UK. (2015). MAHUffe 1.9.0.3. Retrieved from http://www.mrc-epid.cam.ac.uk/physical-activitydownloads

Mitchell, L.E., Ziviani, J., \& Boyd, R.N. (2015a). Characteristics associated with physical activity among independently ambulant children and adolescents with unilateral cerebral palsy. Developmental Medicine \& Child Neurology, 57, 167-174. doi:10.1111/dmen.12560

Mitchell, L.E., Ziviani, J., \& Boyd, R.N. (2015b). Habitual physical activity of independently ambulant children and adolescents with cerebral palsy: Are they doing enough? Physical Therapy, 95, 202-211. doi:10.2522/ptj.20140031

Nilsson, A., Ekelund, U., Yngve, A., \& Sjöström, M. (2002). Assessing physical activity among children with accelerometers using different time sampling intervals and placements. Pediatric Exercise Science, 14, 87-96. doi:10.1123/pes.14.1.87

Nooijen, C.F., Slaman, J., Stam, H.J., Roebroeck, M.E., \& Berg-Emons, R.J. (2014). Inactive and sedentary lifestyles amongst ambulatory adolescents and young adults with cerebral palsy. Journal of Neuroengineering and Rehabilitation, 11, 49. PubMed doi:10.1186/1743-0003-11-49

Obeid, J., Balemans, A.C., Noorduyn, S.G., Gorter, J.W., \& Timmons, B.W. (2014). Objectively measured sedentary time in youth with cerebral palsy compared with age-, sex-, and season-matched youth who are developing typically: An explorative study. Physical Therapy, 94, 1163-1167. PubMed doi:10.2522/ptj.20130333

Pan, C.-Y., \& Frey, G.C. (2005). Identifying physical activity determinants in youth with autism spectrum disorders. Journal of Physical Activity \& Health, 2, 412-422. doi:10.1123/jpah.2.4.412

Pan, C.-Y., \& Frey, G.C. (2006). Physical activity patterns in youth with autism spectrum disorders. Journal of Autism and Developmental Disorders, 36, 597-606. PubMed doi:10.1007/s10803-006-0101-6

Phillips, A.C., \& Holland, A.J. (2011). Assessment of objectively measured physical activity levels in individuals with intellectual disabilities with and without Down's syndrome. PLoS ONE, 6, e28618. PubMed doi:10.1371/journal.pone.0028618

Reilly, J.J., Penpraze, V., Hislop, J., Davies, G., Grant, S., \& Paton, J.Y. (2008). Objective measurement of physical activity and sedentary behaviour: Review with new data. Archives of Disease in Childhood, 93, 614-619. PubMed doi:10.1136/ adc. 2007.133272

Riddoch, C.J., Andersen, L.B., Wedderkopp, N., Harro, M., Klasson-Heggebø, L., Sardinha, L.B., ... Ekelund, U. (2004). Physical activity levels and patterns of 9- and 15-yr-old European children. Medicine \& Science in Sports \& Exercise, 36, 86-92. PubMed doi:10.1249/01.MSS.0000106174.43932.92

Roberts, D.E. (2007). Measurement of physical activity with accelerometers in children. (Electronic Doctoral dissertations, University of Massachusetts Amherst). Retrieved from http://scholarworks.umass.edu/dissertations/AAI3254924 
Rosser Sandt, D.D., \& Frey, G.C. (2005). Comparison of physical activity levels between children with and without autistic spectrum disorders. Adapted Physical Activity Quarterly, 22, 146-159. doi:10.1123/apaq.22.2.146

Rowlands, A.V., Powell, S.M., Humphries, R., \& Eston, R.G. (2006). The effect of accelerometer epoch on physical activity output measures. Journal of Exercise Science \& Fitness, 4, 52-58.

Shields, N., Dodd, K.J., \& Abblitt, C. (2009). Do children with Down syndrome perform sufficient physical activity to maintain good health? A pilot study. Adapted Physical Activity Quarterly, 26, 307-320. PubMed doi:10.1123/apaq.26.4.307

Sit, C.H.P., Lindner, K.J., \& Sherrill, C. (2002). Sport participation of Hong Kong Chinese children with disabilities in special schools. Adapted Physical Activity Quarterly, 19, 453. PubMed doi:10.1123/apaq.19.4.453

Strong, W.B., Malina, R.M., Blimkie, C.J., Daniels, S.R., Dishman, R.K., Gutin, B., ... Trudeau, F. (2005). Evidence based physical activity for school-age youth. The Journal of Pediatrics, 146, 732-737. PubMed doi:10.1016/j.jpeds.2005.01.055

Suzuki, M., Saitoh, S., Tasaki, Y., Shimomura, Y., Makishima, R., \& Hosoya, N. (1991). Nutritional status and daily physical activity of handicapped students in Tokyo metropolitan schools for deaf, blind, mentally retarded, and physically handicapped individuals. American Journal of Clinical Nutrition, 54, 1101-1111. PubMed

Telama, R., Yang, X., Viikari, J., Valimaki, I., Wanne, O., \& Raitakari, O. (2005). Physical activity from childhood to adulthood: A 21-year tracking study. American Journal of Preventive Medicine, 28, 267-273. PubMed doi:10.1016/j.amepre.2004.12.003

Tremblay, M.S., LeBlanc, A.G., Kho, M.E., Saunders, T.J., Larouche, R., Colley, R.C., .. . Connor Gorber, S. (2011). Systematic review of sedentary behaviour and health indicators in school-aged children and youth. International Journal of Behavioral Nutrition and Physical Activity, 8, 98. PubMed doi:10.1186/1479-5868-8-98

Trost, S.G. (2007). State of the art reviews: Measurement of physical activity in children and adolescents. American Journal of Lifestyle Medicine, 1, 299-314. doi:10.1177/ 1559827607301686

Trost, S.G., Pate, R.R., Freedson, P.S., Sallis, J.F., \& Taylor, W.C. (2000). Using objective physical activity measures with youth: How many days of monitoring are needed? Medicine \& Science in Sports \& Exercise, 32, 426-431. PubMed doi:10.1097/ 00005768-200002000-00025

Trost, S.G., Pate, R.R., Sallis, J.F., Freedson, P.S., Taylor, W.C., Dowda, M., \& Sirard, J. (2002). Age and gender differences in objectively measured physical activity in youth. Medicine \& Science in Sports \& Exercise, 34, 350-355. PubMed doi:10.1097/ 00005768-200202000-00025

Tyler, K., MacDonald, M., \& Menear, K. (2014). Physical activity and physical fitness of school-aged children and youth with autism spectrum disorders. Autism Research and Treatment, 2014, 312163. PubMed doi:10.1155/2014/312163

Wennlöf, A.H., Yngve, A., \& Sjöström, M. (2003). Sampling procedure, participation rates and representativeness in the Swedish part of the European Youth Heart Study (EYHS). Public Health Nutrition, 6, 291-299. PubMed doi:10.1079/ PHN2002425

Whitt-Glover, M.C., O’Neill, K.L., \& Stettler, N. (2006). Physical activity patterns in children with and without Down syndrome. Pediatric Rehabilitation, 9, 158-164. PubMed doi:10.1080/13638490500353202

World Health Organization. (2010). Global recommendations on physical activity for health. Geneva, Switzerland: Author.

Yngve, A., Nilsson, A., Sjostrom, M., \& Ekelund, U. (2003). Effect of monitor placement and of activity setting on the MTI accelerometer output. Medicine \& Science in Sports \& Exercise, 35, 320-326. PubMed doi:10.1249/01.mss.0000048829.75758.a0 